\title{
Síndrome del edificio enfermo en los bloques académicos de una institución de educación superior
}

\section{(Sick building syndrome in blocks of an academic institution of higher education)}

\author{
Fabián Ortiz Terán ${ }^{1}$, Bolívar Haro Haro ${ }^{1}$
}

\begin{abstract}
Resumen:
El presente trabajo de investigación tiene el objetivo de caracterizar el Síndrome del Edificio Enfermo (SEE), en tres edificaciones de la Facultad de Ciencias de la Ingeniería de la Universidad Tecnológica Equinoccial, en el campus Occidental. Realizado un análisis comparativo entre los resultados de prevalencia, obtenidos en los análisis de la investigación y comparados con el permisible de la norma NTP 290, se pudo determinar que sí existe el síndrome en los edificios que son sujeto de esta investigación. Se aplicó el cuestionario para los riesgos físicos y para el universo de la investigación $(\mathrm{N}=64)$. Con los resultados y la valoración de la prevalencia, se elaboró una propuesta para el mejoramiento de las condiciones de trabajo de los mismos. La investigación que se presenta servirá como indicador de la calidad de las edificaciones al servicio de la Universidad y para futuras investigaciones.
\end{abstract}

Palabras clave: síndrome; ruido; prevalencia; ventilación; enfermo.

\begin{abstract}
:
This research focusses on characterizing the sick building syndrome (SBS) on three buildings of the Engineering Sciences Faculty, at the Occidental Campus of the Universidad Tecnológica Equinoccial. By means of a comparative analysis between the prevalence result obtained within the research work and a comparison with the allowable levels according to NTP290 Spanish Code, determination was made that, in effect SBS exists in the studied buildings. The physical risk questionnaire was applied to the universe of studied people $(\mathrm{N}=64)$. Based on the results and in accordance to the prevalence appraisal, a proposal was formulated for the remediation of the involved working conditions on the studied buildings. This research will serve as an institutional reference for the building quality indicator of the University facilities and also for future researches.
\end{abstract}

Keywords: syndrome; noise; prevalence; ventilation; sick.

\section{Introducción}

El Síndrome del Edificio Enfermo (SEE) es el nombre que se da al conjunto de síntomas diversos que se presentan predominantemente en los individuos que ocupan estos edificios y que no van generalmente acompañados de ninguna lesión orgánica o signo físico, diagnosticándose, a menudo, por exclusión. (Wriu, 2007). Los factores de riesgo que los originan son múltiples: físicos, químicos, microbiológicos, contaminantes del exterior y factores psicosociales. (Rodríguez y Alonzo, 2004)

\footnotetext{
${ }^{1}$ Universidad Tecnológica Equinoccial, Quito - Ecuador (\{fortiz, bharo\}@ute.edu.ec)
} 
Los edificios enfermos se dan por un conjunto de procesos en los que destacan: malos diseños arquitectónicos, remodelaciones y adecuaciones que no fueron planificadas y que son el resultado del crecimiento de las instituciones de educación superior produciendo el síndrome.

Actualmente pasamos un alto porcentaje de nuestro tiempo en ambientes cerrados, ya sea en el trabajo, en nuestro hogar o en nuestro tiempo libre (centros comerciales, bares, cines, etc.). Esta forma de vida en espacios interiores ha motivado para que la calidad del aire interior sea de interés general y consecuentemente de interés laboral. En ocasiones, este aire no reúne las condiciones idóneas para la salud y es necesario conocer sus características para tratarlo, buscando prevenir y evitar futuros problemas laborales, malas condiciones de trabajo, bajas laborales, etc.

El síndrome del edificio enfermo (SEE) o el sick building syndrome (SBS) fue reconocido como enfermedad por la Organización Mundial de la Salud en 1982. Es un padecimiento muy antiguo del que poco se sabe en los actuales momentos y, que afecta entre un $10 \%$ y un $30 \%$ a los ocupantes de los edificios modernos, es decir, uno de cada tres edificios construidos entre 1965 y 1975 y, que pueden influir apreciablemente en los índices de ausentismo (Berenguer Subils, 2012). Para la Facultad de Medicina de la Universidad Nacional Autónoma de México, el SEE es el nombre que se da al conjunto de varios síntomas que presentan el $20 \%$ aproximadamente de los usuarios de inmuebles generalmente equipados con aire acondicionado. Los síntomas que ocasionan en la salud el trabajar en un edificio enfermo son por lo general leves, aunque a menudo, son causantes de ausentismo, lo que genera un alto costo para las empresas. (Soberanes, 2011).

Según la Asociación para la Prevención de Accidentes, citado por Rubio (2004), "la evaluación del riesgo consiste en un proceso de aplicación sistemática de métodos capaces de identificarlo, valorarlo, actuar sobre él para controlarlo y hacer un seguimiento para poder priorizar la actuación y la efectividad de los resultados de la misma" (p. 18).

EI SEE ocurre cuando se produce una concentración de compuestos contaminantes causados por la falta de regeneración del aire procedente del exterior. Entre estos síntomas se pueden mencionar: irritación y resequedad de oídos, nariz y garganta, que a su vez, generan trastornos en los sentidos del gusto y del olfato.

Las sociedades pasan por fases pre lógicas y supersticiosas en sus explicaciones de las causas de los daños y las desgracias; por lo que, no es pequeño el camino recorrido cuando, abandonados ya los atavismos y los fatalismos de todo tipo, se puede planear la acción preventiva. (Fernando, 2011). 
Por los problemas antes expuestos, el objetivo de esta investigación se refiere a caracterizar el síndrome del edificio enfermo en los bloques académicos de la Facultad de Ciencias de la Ingeniería de la Universidad Tecnológica Equinoccial.

\section{Materiales y Métodos}

A continuación esbozamos los principales materiales y métodos que permitieron la realización de esta investigación, basados en una concepción dialéctica materialista en cuanto a su historicidad, multidimensionalidad y en su carácter sistémico. Por lo que podemos afirmar:

El diseño de esta investigación es no experimental porque se describen los hechos como son observados en la práctica. De cohorte transversal porque se recopilan los datos e información en un solo período de tiempo.

La entrevista, además de obtener una prueba añadida sobre la documentación previamente analizada, corrobora su aplicación a través de los sujetos que la constituyen, en última instancia, los receptores de las medidas preventivas en la empresa y que conocen su efectiva realización. (Nieto Millán, 2005).

El gobierno de España a través del Ministerio de Empleo y Seguridad Social, el Instituto Nacional de Seguridad e Higiene del Trabajo (INSHT), generan notas técnicas de prevención con la finalidad de dar una herramienta indispensable para todo prevencionista $y$, una de ellas es la norma NTP 290 (Solé y Pérez, sf).

EI INSHT, es el organismo de referencia nacional de España en materia de seguridad y salud en el trabajo, que ejerce la Secretaría de la Comisión Nacional de Seguridad y Salud en el Trabajo y actúa de centro de referencia nacional en relación con las instituciones de la Unión Europea (Sánchez 2011).

Las pautas generales para la identificación del síndrome del edificio enfermo están en la NTP 290 y son:

- En el edificio (su edad, los materiales empleados, las obras y/o remodelaciones realizadas).

- Los ocupantes (su número y distribución en el edificio).

- Los materiales y equipos de trabajo (naturaleza y ubicación).

- El sistema de climatización / ventilación.

\section{Comprobación de Proposiciones}

En esta parte de la investigación se estableció la comprobación de las proposiciones, a través de las preguntas científicas, las cuales constituyeron la guía para resolver la problemática planteada. 
1. ¿Cómo identificar las condiciones generales para determinar un edificio enfermo?

Para resolver esta interrogante se procedió a estudiar las teorías del síndrome del edificio enfermo, teniendo como normativa a utilizar la NTP 290, donde se establecen las condiciones generales para determinar un edificio enfermo según su cuestionario y parámetros para su evaluación.

2. ¿Cómo realizar una descripción de ventilaciones, superficies interiores, materiales de construcción y hermeticidad que permite el desarrollo del edificio enfermo?

El cuestionario permite detectar a través de sus preguntas el SEE, debido al grado de satisfacción de los usuarios de los edificios.

3. ¿Cómo evaluar por medio de check list la presencia del síndrome del edificio enfermo?

Se recogieron los criterios de los encuestados y posteriormente se llevaron a la escala de evaluación de la norma, en la que si la prevalencia es mayor o igual a 0.20 o el $20 \%$, se establece que si hay el síndrome del edificio enfermo y así lo demostró está investigación, que hay síndrome del edificio enfermo en los tres bloques estudiados.

4. ¿Cómo verificar los desencadenantes del síndrome del edificio enfermo tomando en cuenta la calidad del aire, la cantidad y calidad de temperatura, humedad, iluminación y ruido?

Estableciendo los criterios con respecto a las áreas seleccionadas y teniendo en cuenta las teorías estudiadas, se establecieron los desencadenantes por calidad del aire, debido a que nos encontramos en presencia de edificios muy cerrados y pueden causar enfermedades respiratorias, por lo tanto la temperatura no es la ideal y se experimentan cambios dentro del edificio.

5. ¿Cómo identificar las condiciones generales para determinar un edificio enfermo?

Para resolver esta interrogante se procedió a estudiar la teoría del síndrome del edificio enfermo, desde su surgimiento, antecedentes, principales teorías, modelo, normas internacionales y, partiendo de esto se estableció el estado del arte del objeto de estudio, teniendo como normativa a la NTP 290, donde se establecen las condiciones generales para determinar un edificio enfermo según su cuestionario y parámetros para su evaluación.

6. ¿Cómo describir la ventilación, superficies interiores, materiales de construcción y hermeticidad que permite el desarrollo del edificio enfermo?

Para resolver esta interrogante se utilizó la norma NTP 290, en la que dentro de sus interrogantes están comprendidos los aspectos que se plantean en la pregunta y, a través del cuestionario se 
preguntó a los trabajadores de los bloques en cuestión, sus criterios con respecto a la ventilación y las condiciones en las que se encuentran.

7. ¿Cómo evaluar por medio de check list la presencia del síndrome del edificio enfermo?

Se recogieron los criterios de los encuestados y se llevaron a la escala de evaluación de la norma, donde si la prevalencia es mayor o igual a 0.20 o el $20 \%$, se establece que si hay Síndrome del Edificio Enfermo y así lo demostró está investigación en los tres bloques estudiados.

8. ¿Cómo verificar los desencadenantes del síndrome del edificio enfermo tomando en cuenta la calidad del aire, la cantidad y calidad de temperatura, humedad, iluminación y ruido?

Estableciendo los criterios con respecto a las áreas seleccionadas y teniendo en cuenta las teorías estudiadas, se comprobó los desencadenantes por: calidad del aire, debido a que nos encontramos en presencia de edificios muy cerrados y pueden causar enfermedades respiratorias, por lo tanto la temperatura no es la ideal y se experimentan cambios desde dentro del edificio.

El fin de la investigación una vez determinadas las causas, es implantar las medidas que las corrigen, comprobar su eficacia y mantenerlas (Azcuénaga, 2009).

\section{Resultados}

Los resultados se presentan en las tablas donde están identificados los bloques y la caracterización de los mismos en base a la encuesta realizada. El procedimiento adoptado fue el de la norma NTP 290, que se basa en la prevalencia de los resultados consultados y los límites para determinar si un edificio presenta SEE.

EI SEE presentó los siguientes resultados:

La Tabla 1 y la Tabla 2, presentan las consecuencias y el resumen de la investigación de los tres bloques, respecto a la sintomatología de las edificaciones, donde se muestran los resultados en porcentajes de iluminación, ruido, vibraciones, ambiente térmico/humedad y ventilación.

Tabla 1. Sintomatología en edificación.

\begin{tabular}{lcccc}
\hline & Edificio B Total & Edificio \\
Afectados & Cumple & $\begin{array}{c}\text { Edificio G Total } \\
\text { Afectados }\end{array}$ & Cumple & $\begin{array}{c}\text { Biblioteca Total } \\
\text { Afectados }\end{array}$ \\
\hline Iluminación & $14 \%$ No cumple & $25 \%$ Cumple & $9 \%$ No cumple \\
Ruido & $60 \%$ Cumple & $40 \%$ Cumple & $30 \%$ Cumple \\
Vibraciones & 0 No cumple & 0 No cumple & 0 No cumple \\
Ambiente Térmico/Humedad & $30 \%$ Cumple & $47 \%$ Cumple & $31 \%$ Cumple \\
Ventilación & $19 \%$ No cumple & $63 \%$ Cumple & $19 \%$ No cumple \\
\hline
\end{tabular}


Tabla 2. Resumen total de la investigación - sintomatología en edificación.

\begin{tabular}{ccc}
\hline Afectación por Edificio & Porcentaje & Diagnóstico \\
\hline Edificio B Total Afectados & $60 \%$ SEE Confirmado \\
Edificio G Total Afectados & $63 \%$ SEE Confirmado \\
Edificio Biblioteca Total Afectados & $31 \%$ SEE Confirmado \\
\hline
\end{tabular}

En la Tabla 3 y Figura 1, se muestra el resultado de confort que presenta el puesto de trabajo al estar junto a una ventana, obteniendo como resultado que los tres edificios en estudio tienen disconfort en determinadas áreas de trabajo, activándose el indicador del SEE. La molestia de no tener luz natural y la satisfacción de zonas de calor natural producido por los rayos solares, inducen a los trabajadores a salir a lugares de mayor temperatura en determinados momentos de su labor.

Tabla 3. Sitio donde labora.

Resultados de los bloques B, G, Biblioteca - tutores distancia, del sitio de trabajo a una ventana

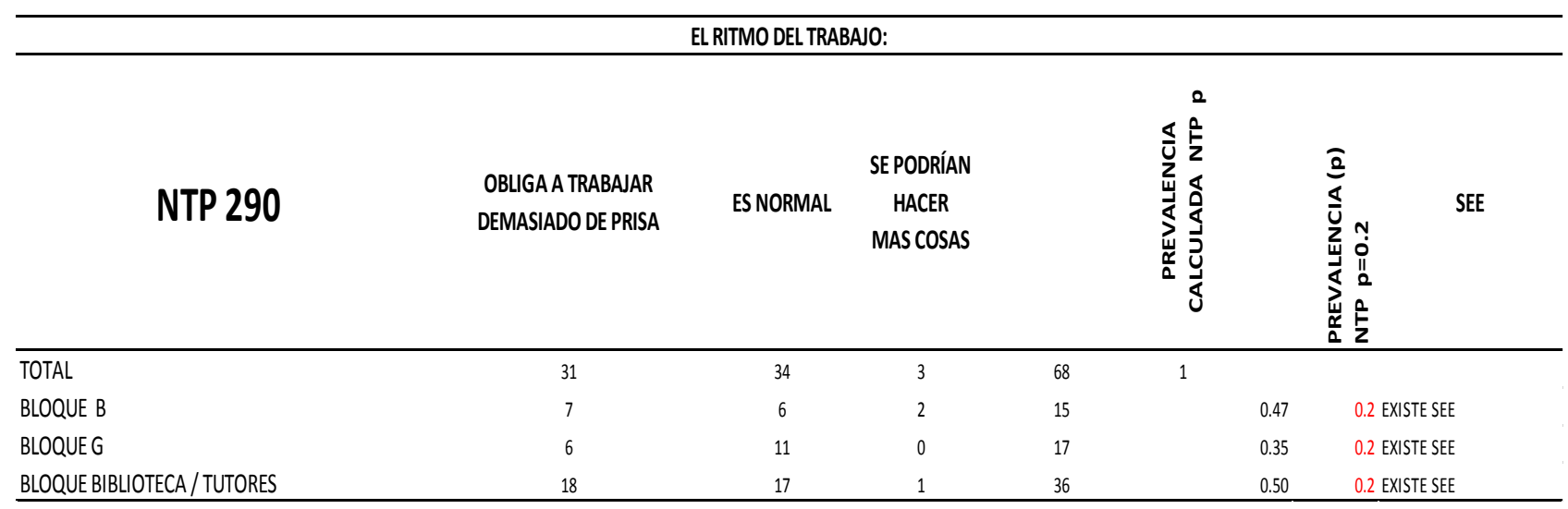
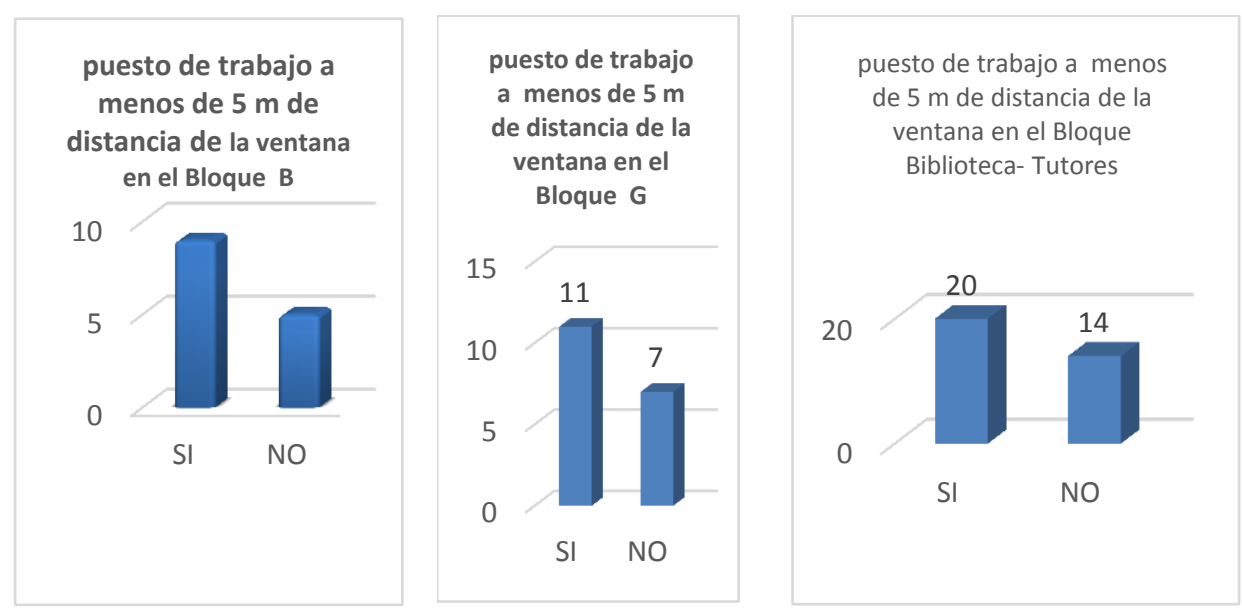

Figura 1. Ubicación de sitio de trabajo con respecto a la ventana en los edificios

De la matriz de resultados (Tabla 4 y Figura 2), se puede obtener que en los tres edificios se activa el indicador del SEE, esto significa que la prevalencia de acuerdo a la NTP 290, detecta que el parámetro supera lo permisible y en consecuencia se presume que los edificios tienen síntomas del edificio enfermo. 
En el Bloque B, las conversaciones en los ambientes de trabajo son lo que más incomodan a los trabajadores, porque su trabajo requiere de concentración. También los equipos de oficina son una molestia cuando están dañados o deteriorados, porque producen ruido. En el bloque G, la presencia de los equipos de laboratorio son perjudiciales en esas áreas de trabajo, en el de Bibliotecas y tutorías se escucha un ruido producido desde el exterior, a causa de los estudiantes ingresando a la biblioteca y en especial la cercanía del bloque al patio de mantenimiento de la empresa de basura del Distrito Metropolitano de Quito.

Tabla 4. Resultados de los bloques B, G, Biblioteca -Tutores: ruido

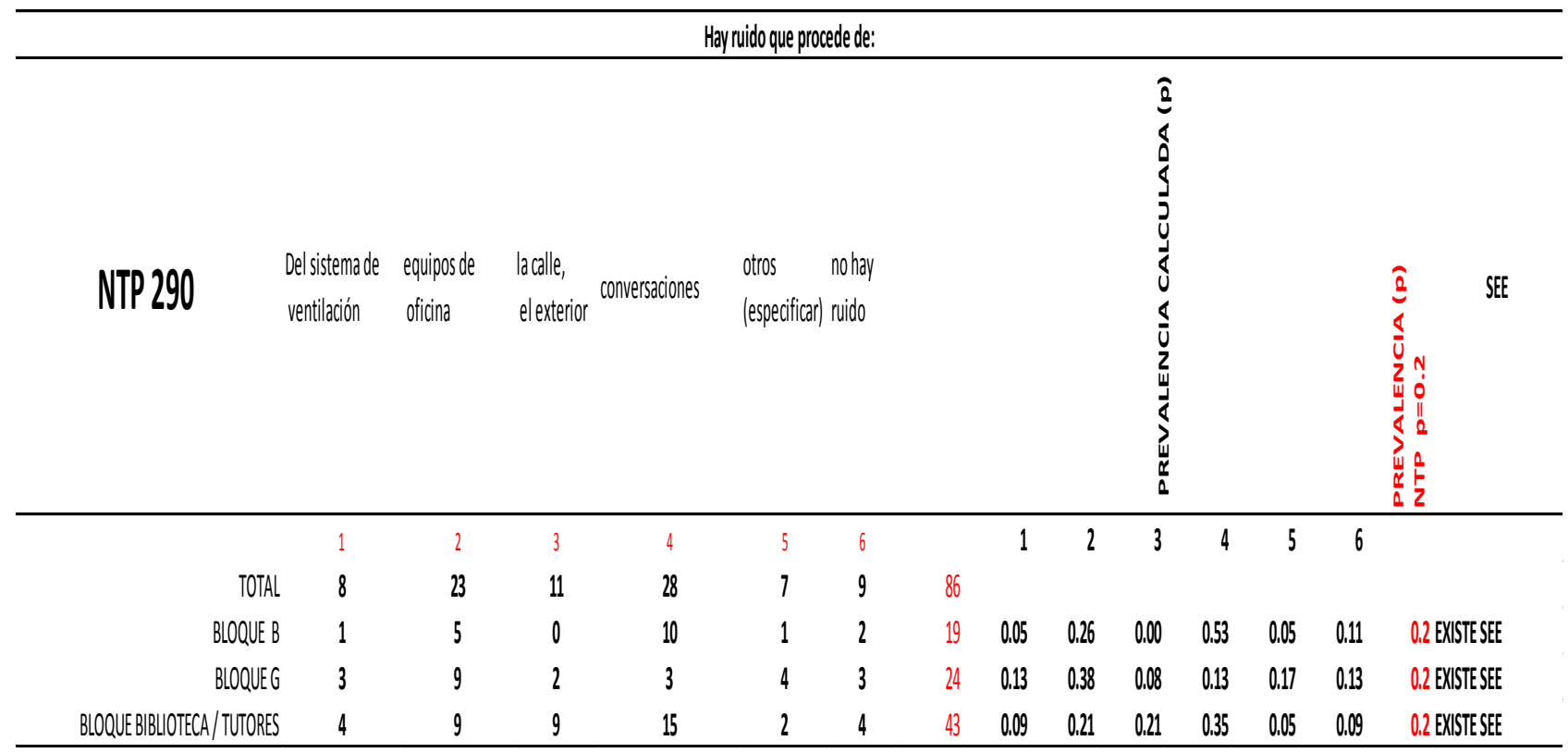
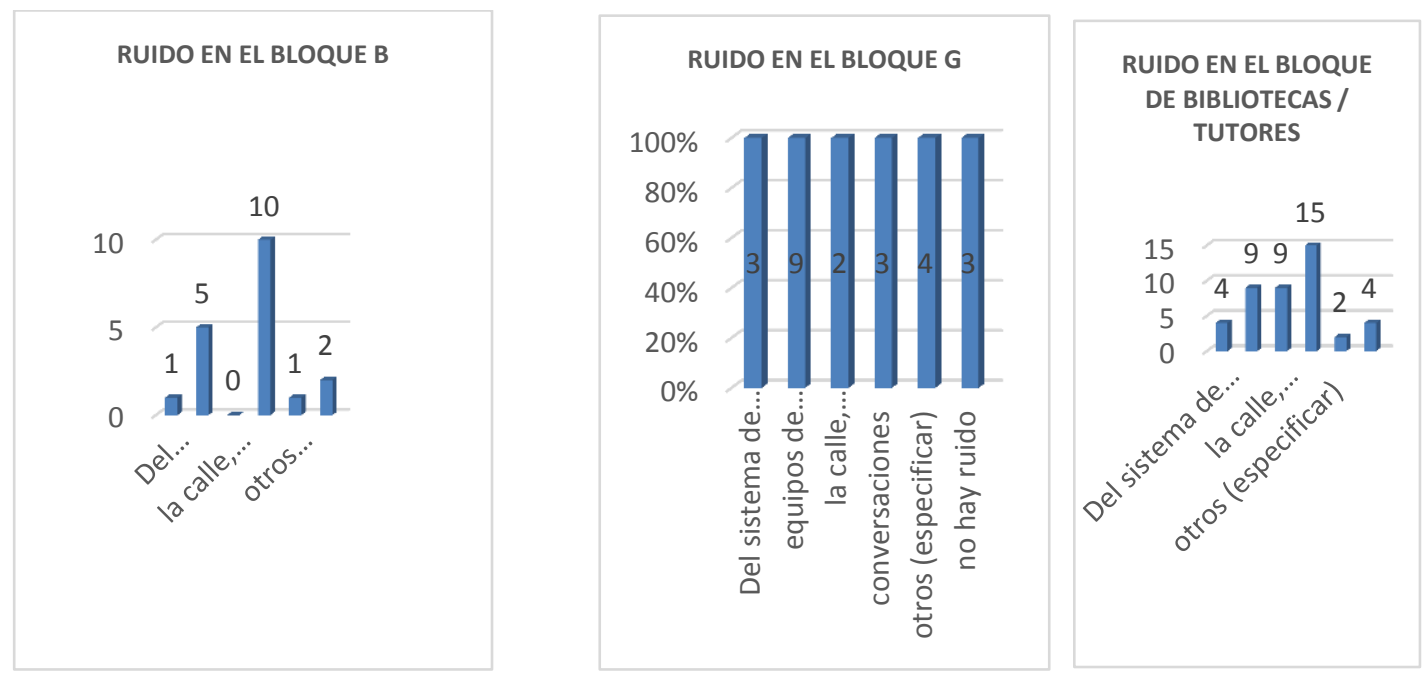

Figura 2. Procedencia del ruido en los edificios

El resultado de la investigación respecto a la temperatura (Tabla 5 y Figura 3), determinó que son bajas, especialmente en las horas pico, por el tipo de materiales empleados en la construcción y en acabados, los cuales colaboran para que la temperatura baje en los tres bloques de la investigación. El indicador se activa en el bloque de bibliotecas, con el problema 
del calor, por ser un sitio donde se concentran los estudiantes a las consultas e investigación y debido a que el sistema de aire acondicionado no funciona.

Tabla 5. Resultados de los Bloques B, G, Biblioteca - Tutores: temperatura/humedad LA TEMPERATURA/HUMEDAD PRODUCE:

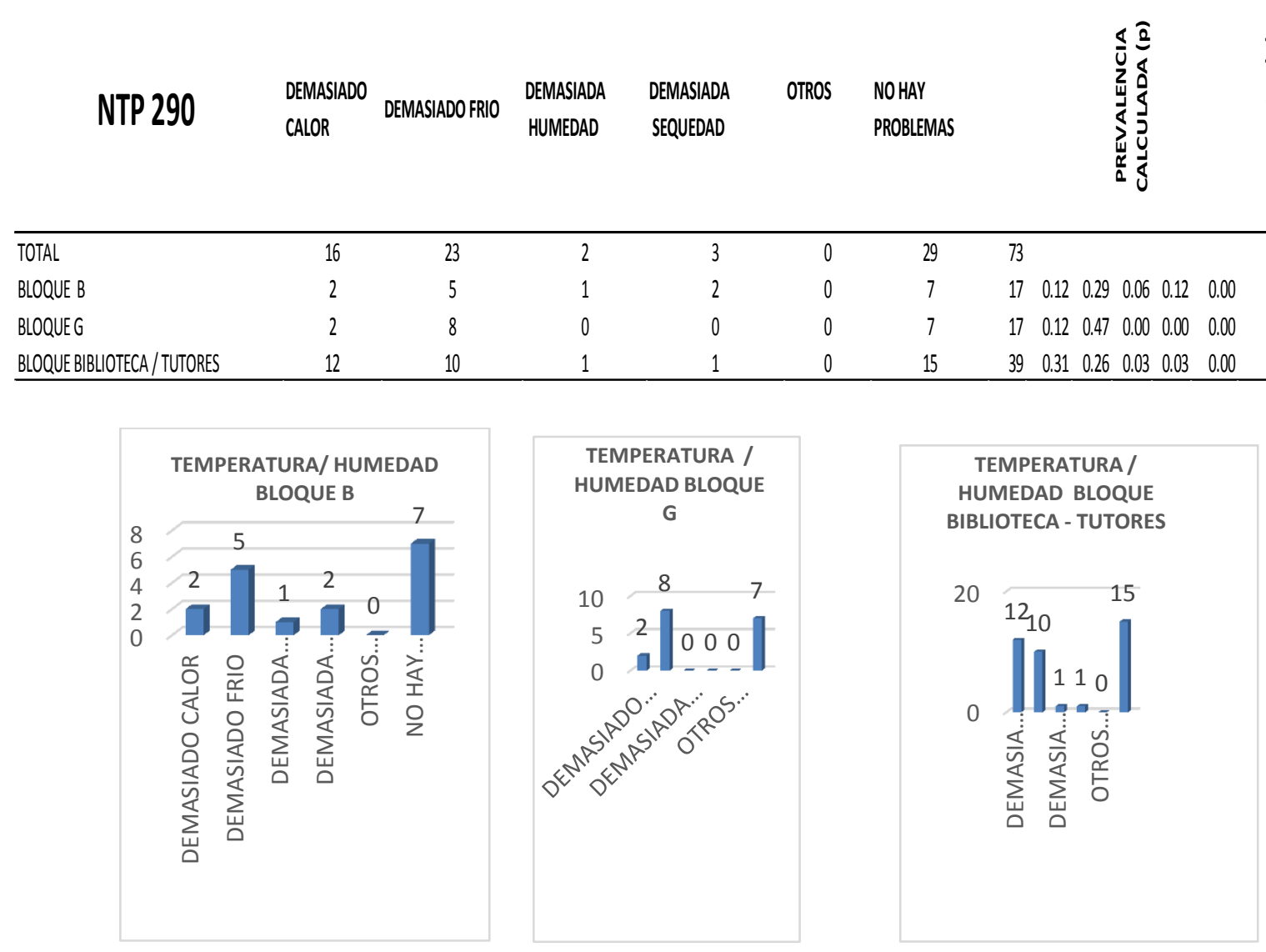

Figura 3. Caracteristicas de temperatura/humedad en los edificios

\section{Discusión}

El estudio permitió caracterizar el síndrome del edificio enfermo (SEE) en las tres construcciones de la Facultad de Ciencias de la Ingeniería (Bloque B, Bloque G y Biblioteca- Sala de tutores), con la herramienta utilizada por la NTP 290, que se basa en la prevalencia en función del cuestionario diseñado para el efecto.

El $77 \%$ de los ocupantes de los 3 bloques se encuentran en el rango de mayores de 30 años. El $63 \%$ es de género masculino y el $37 \%$ de género femenino.

En las tres edificaciones se evidenció la presencia del SEE por diferentes causas. Las características de cada bloque por su funcionabilidad dieron como resultado diferentes factores de prevalencia significativa; en el bloque $B$, la adaptación de nuevos diseños y remodelaciones afectaron el confort, la iluminación, el ruido producido por los visitantes en las oficinas, etc.

En el bloque G, se pudo detectar que no fue diseñado para tantos laboratorios y se readecuó, afectando la ventilación, iluminación y temperatura. 
El bloque de Biblioteca, es un galpón que se adecuó para el funcionamiento de la biblioteca y sala de tutores, con el gran problema de olores producidos en el exterior por la presencia del taller de los carros de la basura del Distrito Metropolitano de Quito.

De la matriz de resultados se concluyó que en los tres edificios se activa el indicador del SEE, esto significa que la prevalencia de acuerdo a la NTP 290, detecta que el parámetro supera lo permisible y en consecuencia se presume que los edificios tienen síntomas del edificio enfermo.

En el edificio $B$, las conversaciones en los ambientes de trabajo son las que más molestan a los trabajadores porque su actividad requiere de concentración, también los equipos de oficina son incómodos cuando están dañados o deteriorados ya que generan ruido.

En el Bloque G, los equipos de laboratorios, talleres, planta de alimentos son los que causan el SEE.

En el Bloque de Bibliotecas y Tutorías se presenta un ruido que proviene del exterior, que es de los estudiantes ingresando a la biblioteca y en especial la cercanía del bloque al patio de mantenimiento de la empresa de basura del Distrito Metropolitano.

\section{Conclusiones y recomendaciones}

Por medio de la utilización del método de la NTP 290 se pudo establecer que los 3 edificios padecen del SEE.

Los resultados obtenidos en las encuestas indicaron una importante presencia de prevalencias que activaron los indicadores del SEE.

Las adecuaciones no planificadas producen espacios físicos, que por sus características, producen el síndrome del edificio enfermo.

Los edificios con poco mantenimiento, se deterioran, provocando daños en los equipos de ventilación y esto desencadena factores que permiten la presencia del SEE.

Los materiales utilizados en acabados son otro factor que incomoda a los ocupantes del edificio, dando como consecuencia, malestares que se presentan con alergias, resfriados, dolores de cabeza y que desaparecen una vez que abandonan los edificios.

El estudio realizado puede servir como el principio de otras investigaciones para llegar a minimizar el SEE.

Se recomienda que en las futuras construcciones y adecuaciones prevalezca la planificación, porque los cambios de diseño han provocado sin duda la aparición del SEE. 
El bloque B, presenta el agrupamiento de áreas sin ventanas y con permanente luz artificial, que por el espacio limitado que disponen, no permiten el desenvolvimiento de las tareas con normalidad, teniendo complicaciones con el olor, temperatura y ventilación.

El bloque $\mathrm{G}$, es el que presenta problemas de olores y ventilación; se recomienda la reubicación en otro bloque, por lo menos el 50 \% de los laboratorios o mejorar el sistema de ventilación.

El bloque de Bibliotecas y la sala de Tutores, deben ser reubicados fuera del radio de olor y con una infraestructura diseñada para el efecto.

El tipo de materiales usados en el área administrativa debe adecuarse con materiales térmicos.

\section{Bibliografía}

Azcuénaga Lizana, L. (2009). Manual práctico para la investigación de accidentes e incidentes laborales (3. ${ }^{\mathrm{a}}$ ed.). Madrid: FC Editorial.

Berenguer Subils, (2012). Síndrome del edificio enfermo. Madrid: FundaciónMapfre

Fernando Pablo, J. et al. (2011). Manual de seguridad en el trabajo (2. ${ }^{a}$ ed.). Madrid: Fundación MAPFRE.

Nieto Millán, J. et al. (2005). Manual de coordinación de seguridad y salud en obras de construcción. Madrid: Ecoiuris.

Rodríguez Hernández, L. y Alonzo Salomón, J. (2004). Efecto de los factores ambientales, laborales y psicosociales, en el síndrome del edificio enfermo. Ingeniería, 2(8), pp. 1-8.

Rubio Romero, J. C. (2004). Métodos de evaluación de riesgos laborales. Madrid: Ediciones Díaz de Santos.

Sánchez Rivero, J., Palomino Márquez, T., González Barriga, J. y Tejada Montesinos, J. (2011). El coordinador de seguridad y salud (3. ${ }^{a}$ ed.). Madrid: FC Editorial.

Soberanes Collado, Ma. (2011). Síndrome del edificio enfermo. Recuperado de https://esdaiposgrados.wordpress.com/page/4/?app-download=nokia.pdf

Solé Gómez, Ma. D. y Pérez Nicolás, J. (s.f.). NTP 290: El síndrome del edificio enfermo: cuestionario para su detección. Recuperado de http://www.insht.es/inshtweb/contenidos /documentacion/fichastecnicas/ntp/ficheros/201a300/ntp_290.pdf

Wriu Valenzuela, P. (2007). Síndrome del edificio enfermo. Recuperado de http://www.ergocupacional.com/4910/58976.html 